\title{
EDITORIAL
}

\section{WHY and HOW to deepen education of Evidence-based Practice?}

The problematics of Evidence-Based Practice (EBP) are not granted enough space either in the education of succeeding generations of professionals in nursing and midwifery, or in the education of prospective teachers of health-related subjects. Consequently, we often fail to appreciate its importance. Our professional community has only a partial awareness of the fact that Evidence-Based Practice (EBP) offers a deeply thoughtful way of working with information, and, simultaneously, leads to responsible decision-making by professionals in specific situations in their working life. The main goal of EBP is to continuously improve the activities which individual disciplines focus on, and to support professionals in choosing the best methods or procedures, from several options, in order to solve a particular problem. The methodology of EBP was primarily developed for medicine. Nowadays, it is a given fact that the methodological rules of EBP are applicable in almost all scientific disciplines: nursing, midwifery, pedagogy, sociology, and psychology, among others. Since EBP covers a broad set of processes in which the application of specific tools and methods is necessary, I consider extension and deepening of education in this field to be essential.

Building on my experience acquired through ten years of cooperation with highly erudite EBP experts, and through my work in the Centre of Excellence within the international network of the Joanna Briggs Institute, I would like to put forward some suggestions. First, the curricula of tertiary education study programmes should involve EBP subjects from the first year of bachelor courses, and this should continue through master degree study. The problematics of EBP should be taught by well-trained teachers who have previously had research experience. It is indispensable that these teachers have the requisite language skills [i.e., English to at least $\mathrm{B} 2 / \mathrm{C} 1$ level of the Common European Framework of Reference for Languages (CEFR)]. Teachers should have completed specialised EBP courses and they should be willing to adapt their own existing methods of working with professional information to EBP methodology. Optimally, the trained teachers should continuously cooperate with some of the Evidence-Based Healthcare (EBHC) centres of the Joanna Briggs Institute network. Under such conditions, they have access to the standard tools which they need for high quality EBP teaching, and can hone the expertise acquired from training courses. Teachers should further improve their knowledge and skills by joining scientific teams responsible for the creation of registered systematic reviews that make recommendations for professional practice.

In connection with the need to boost the quality of education and healthcare, theoretical and practical knowledge of EBP is becoming the cornerstone of further improvement in both pedagogy and health. Therefore, I recommend that bachelor's and master's curricula include a special EBP module, focusing on several subjects. The study of EBP should start from the first year of study. The subjects of this module should continue throughout the years of study, and should include a significant practical component. On completing their studies, students should be deeply familiar with the methods and procedures of each EBP phase. This means that graduates should be able to search for and classify information in order to solve a specific problem, to then critically evaluate it, and make responsible decisions about its possible use in practice.

Assoc. Prof. Jana Marečková, Ph.D. Adjunct Associate Professor of The School of Public Health, Adelaide University Department of Anthropology and Health Education Faculty of Education, Palacký University Olomouc 